\title{
Chalcogenide Waveguides for Mid-Infrared Biomedical Sensing Applications
}

\author{
Ganapathy Senthil Murugan, Vinita Mittal, Chris Craig, Dan Hewak and James S Wilkinson \\ Optoelectronics Research Centre, University of Southampton \\ Southampton, United Kingdom \\ smg@orc.soton.ac.uk
}

\begin{abstract}
High contrast $\mathrm{GeTe}_{4}$ channel waveguides were fabricated on silicon with a $\mathrm{ZnSe}$ isolation layer using the lift-off technique. Waveguiding in these channels was demonstrated in the mid-wave and long-wave infrared spectral regions. The results of numerical modelling on the optical loss with different isolation layer thicknesses are also briefly discussed.
\end{abstract}

Keywords-Mid-IR Waveguide; chalcogenide; biosensing

\section{INTRODUCTION}

Mid-infrared absorption spectroscopy is the most widely used technique for detecting the molecular signatures of an unknown sample using their fingerprint absorption. The evanescent field at the waveguide-air interface is used to produce the spectrum of a sample in contact with the waveguide. This is highly sensitive technique for biosensing and requires only a very small sample volume. Performing waveguide evanescent spectroscopy in the mid-IR spectral region $(2-14 \mu \mathrm{m})$ will be useful in avoiding recognition labels during sensing allowing direct detection of analytes. Waveguides fabricated out of conventional materials such as silica and other oxides stop transmitting beyond 3-5 $\mu \mathrm{m}$. However, chalcogenide materials transmit up to about $20 \mu \mathrm{m}$ in the mid-IR. Recently we demonstrated high contrast $\mathrm{GeTe}_{4}$ waveguides fabricated on bulk ZnSe substrates [1] for sensitive detection in the mid-IR spectral region. However, bulk ZnSe substrates presented some challenges in achieving good quality polishing at the waveguide end-facets. Therefore, we have designed $\mathrm{GeTe}_{4}$ waveguides on silicon substrates with $\mathrm{ZnSe}$ isolation layers, which enable very high quality waveguide end-facets and much lower insertion loss. In this paper we present the fabrication and mid-IR characterization results of $\mathrm{GeTe}_{4}$ waveguides on silicon.

\section{DESIGN AND FABRICATION OF WAVEGUIDES}

The optical loss incurred by the fundamental mode of $\mathrm{GeTe}_{4}$ waveguides with dimensions supporting single mode operation on silicon substrate with varying $\mathrm{ZnSe}$ isolation layer thickness was modelled using Comsol. Figure 1(a) shows that at a thickness of $3 \mu \mathrm{m} \mathrm{ZnSe,} \mathrm{the} \mathrm{loss} \mathrm{due} \mathrm{to} \mathrm{the} \mathrm{underlying}$ silicon substrate is much less than $0.1 \mathrm{~dB} / \mathrm{cm}$ which is sufficient for most practical applications. Also note that the higher order modes, if they exist, will incur much higher loss than this for the same isolation layer thickness and therefore, this will also act as higher order mode stripper. We have

This research is funded by the European Research Council under the EU 7th Framework Programme (FP7/2007-2013) / ERC grant agreement no. 291216 deposited $4 \mu \mathrm{m}$ thick films of $\mathrm{ZnSe}$ on silicon substrates using sputtering and then straight channels of $\mathrm{GeTe}_{4}$ were fabricated by patterning a negative photoresist with trenches with desired undercuts, followed by sputter deposition of $\mathrm{GeTe}_{4}$ and finally removal of the resist patterns. Fig. 1(b) shows the SEM crosssectional image of a cleaved end facet of the waveguide.

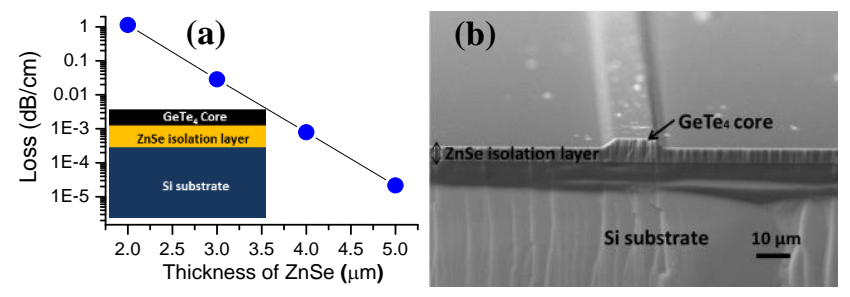

Fig. 1. (a) Waveguide loss vs isolation layer thickness and (b) SEM image of a $\mathrm{GeTe}_{4}$ channel waveguide with $\mathrm{ZnSe}$ isolation layer on Silicon.

\section{WAVEGUIDE CHARACTERISATION}

Light from a tunable OPO $(2.5-3.7 \mu \mathrm{m})$ and QCL $(6-12$ $\mu \mathrm{m})$ was coupled into single mode fibers $\left(\mathrm{ZrF}_{4} / \mathrm{As}_{2} \mathrm{Se}_{3}\right)$ using $\mathrm{ZnSe}$ objective lens. The other end of the fiber was buttcoupled to the $\mathrm{GeTe}_{4}$ waveguides. The guided light emerging from the channel waveguides was imaged from above using thermal imaging cameras (FLIR InSb and microbolometer). Fig. 2 ( $a$ and $b$ ) show the top view of the output end of the waveguides showing light scattered from the end of a channel at a wavelength of $3.7 \mu \mathrm{m}$ and $9 \mu \mathrm{m}$. Near field imaging of the end facets were also carried out using a $\mathrm{ZnSe}$ objective lens to image the actual guided mode.

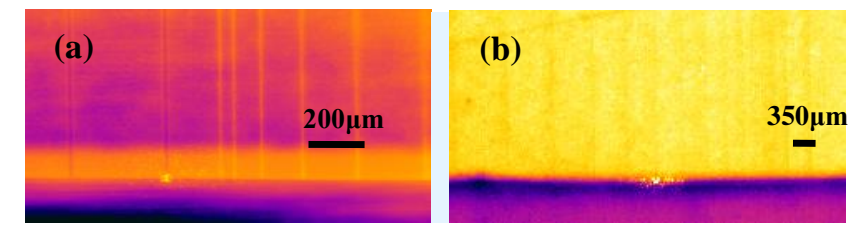

Fig. 2. Images of the guided mode at the end of channel waveguide at (a) $3.7 \mu \mathrm{m}$ and (b) $9 \mu \mathrm{m}$ wavelength.

\section{REFERENCES}

[1] V. Mittal, L. G. Carpenter, J. C. Gates, J. S. Wilkinson, and G. S. Murugan, "GeTe 4 channel waveguides for the mid-wave infrared spectral band," in 12th International Conference on Fiber Optics and Photonics, OSA Technical Digest (2014) , paper T4B.4 